\title{
Produtos naturais de origem vegetal como ferramentas alternativas para o controle larvário de Aedes aegypti e Aedes albopictus
}

\section{Natural plant products as an alternative tool for Aedes aegypti and Aedes albopictus larvae control}

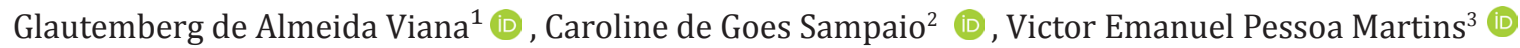

1. Discente do Programa de Pós-graduação em Sociobiodiversidade e Tecnologias Sustentável pela Universidade da Integração Internacional da Lusofonia Afro-brasileira, Redenção, CE, Brasil. 2. Instituto Federal de Educação, Ciência e Tecnologia do Ceará, Campus Maracanaú, CE, Brasil. 3. Docente do Programa de Pós-graduação em Sociobiodiversidade e Tecnologias Sustentável pela Universidade da Integração Internacional da Lusofonia Afro-brasileira, Redenção, CE, Brasil.

\section{Resumo}

Introdução: Aedes aegypti e Aedes albopictus são os principais vetores dos vírus Dengue, Zika e Chikungunya. Extratos vegetais e óleos essenciais vêm sendo utilizados em pesquisas como alternativas aos inseticidas sintéticos tradicionalmente utilizados nos programas de controle dessas doenças. Métodos: de julho a novembro de 2017, foi realizado um levantamento de artigos científicos publicados entre os anos 2000 e 2016 nas bases de dados Scielo, Science Direct e Scopus. Foram utilizados os descritores controlados "culicidae", "óleos essenciais" e "extratos vegetais" com interposição do operador boleano "AND". Resultados: inicialmente, foram obtidos 239 artigos. Dezesseis artigos - cinco apareciam em mais de uma base de dados e 11, cujos conteúdos não estavam disponíveis na íntegra, foram excluídos. Dos 223 artigos resultantes, 112 foram excluídos por contemplarem outras espécies de culicídeos (63) e pela não realização de ensaio larvicida (49). Dos 111 artigos restantes, 42 não apresentavam valores referentes à $\mathrm{CL}_{50}$, resultando em 69 artigos cujos conteúdos foram analisados. Foram identificadas 219 espécies vegetais, pertencentes a 43 famílias botânicas, com destaque para Lamiaceae, Myrtaceae, Rutaceae, Cupressaceae, Asteraceae, Pinaceae e Lauraceae. Os óleos essenciais foram os produtos vegetais mais amplamente testados contra Ae. aegypti (158) e Ae. albopictus (43), bem como os que exibiram maior eficiência na mortalidade das larvas $\left(\mathrm{CL}_{50}<100 \mathrm{ppm}\right)$. Conclusões: a busca por novas estratégias de controle de insetos vetores de patógenos que substituam os inseticidas sintéticos tradicionalmente utilizados vem ganhando destaque. Extratos vegetais e óleos essenciais com efeito larvicida significativo constituem promissoras alternativas ao controle de doenças transmitidas por esses artrópodes.

Palavras-chave: Produtos naturais. Atividade larvicida. Aedes aegypti. Aedes albopictus.

\begin{abstract}
Introduction: PAedes aegypti and Aedes albopictus are the main vectors of Dengue, Zika and Chikungunya viruses. Plant extracts and essential oils have been used as an alternative to synthetic insecticides commonly used in control programmes of these diseases. Methods: From July to November 2017 a survey of scientific articles published between 2000 and 2016 years was carried out in the databases Scielo, Science Direct and Scopus. The descriptors "culicidae", "essential oils" and "vegetal extracts" were used with the input of the boolean operator "AND". Results: A total of 239 articles were obtained. Sixteen articles - 5 that appeared in more than one database and 11 whose contents were not available in full - were initially excluded. Of the 223 resulting articles, 112 were excluded because they included other species of culicidae (63) and the non-performance of larvicidal tests (49). Of the 111 remaining articles, 42 presented no $\mathrm{LC}_{50}$ values, resulting, at the end of the selection, in 69 articles whose contents were analyzed. A total of 219 plant species belonging to 43 botanical families were identified, especially Lamiaceae, Myrtaceae, Rutaceae, Cupressaceae, Asteraceae, Pinaceae and Lauraceae. Essential oils were the most widely tested plant products against Ae. aegypti (158) and Ae. albopictus (43), as well as those that showed greater efficiency in the mortality of the larvae $\left(\mathrm{LC}_{50}<100 \mathrm{ppm}\right)$. Conclusions: The search for new strategies for vector control that replace the traditional insecticides used has been highlighted. Plant extracts and essential oils with pronounced larvicidal effect are promising alternatives to the control of diseases transmitted by these arthropods.
\end{abstract}

Key words: Natural products. Larvicidal activity. Aedes aegypti. Aedes albopictus

\section{INTRODUÇÃO}

Os mosquitos desempenham um papel de destaque no cenário das doenças infectoparasitárias em virtude de sua atuação como vetores de uma grande variedade de organismos patogênicos ${ }^{1}$. Mosquitos do gênero Aedes, particularmente o Aedes aegypti e o Aedes albopictus, responsáveis pela transmissão da Dengue, Zika e Chikungunya, estão intimamente relacionados com episódios epidêmicos dessas arboviroses em diversos países, sobretudo os situados nas zonas tropical e subtropical do planeta 2 .

As dificuldades inerentes à produção de uma vacina realmente eficaz contra os sorotipos circulantes dos vírus Dengue, Zika e Chikungunya intensificaram as ações de combate aos seus vetores como a principal estratégia preventiva ao surgimento 
de novos casos dessas doenças. Durante muitos anos, o controle das populações de Ae. aegypti e Ae. albopictus foi feito por meio do uso de inseticidas sintéticos, tais como os organoclorados, organofosforados e piretroides. Entretanto, o uso frequente e indiscriminado dessas substâncias tem causado poluição ambiental, intoxicação em humanos e outros organismos não alvos e, sobretudo, acelerado o processo de seleção de populações resistentes desses mosquitos ${ }^{3,4}$.

Na última década, pesquisas centradas na utilização de produtos naturais de origem vegetal com ação inseticida aumentaram significativamente, uma vez que são fontes de substâncias bioativas e facilmente biodegradáveis ${ }^{5}$. Nesse sentido, extratos e óleos essenciais obtidos de plantas vêm sendo testados para o controle de insetos em virtude de sua elevada seletividade, degradação a produtos não tóxicos ou de baixa toxicidade a organismos não alvos e ao meio ambiente ${ }^{6}$. Esses produtos, provavelmente, contêm fitoquímicos com ação inseticida, os quais são, predominantemente, metabólitos secundários produzidos em resposta a certas condições ambientais, podendo atuar, virtualmente, em todas as fases do desenvolvimento do inseto, inclusive no estágio adulto ${ }^{7,8,9}$.

Nesse sentido, o objetivo deste estudo foi realizar um levantamento acerca das contribuições científicas produzidas no período compreendido entre os anos 2000 e 2016 em relação à ação de produtos vegetais no controle populacional de Ae. aegypti e Ae. albopictus.

\section{MÉTODOS}

Realizou-se um levantamento de artigos científicos cuja abordagem central estivesse relacionada com o uso de produtos naturais de origem vegetal no controle de populações de culicídeos vetores de patógenos. Para isso, buscaram-se artigos disponibilizados nas bases de dados Scielo, Science Direct e Scopus, publicados no período de 2000 a 2016.

A busca dos artigos foi realizada nos meses de julho a novembro de 2017, por meio de busca avançada nas bases de dados, utilizando-se, como descritores controlados, os termos e as expressões "culicidae", "óleos essenciais" e "extratos vegetais", com a interposição do operador boleano "AND".

A seleção dos artigos pautou-se nos seguintes critérios de inclusão: artigos disponíveis on line na íntegra, nos idiomas português, inglês e espanhol; mosquitos das espécies $A e$. aegypti e/ou Ae. albopictus desafiados ao ensaio larvicida e definição da concentração letal do(s) produto(s) testado(s) que resulta $(\mathrm{m})$ na morte de $50 \%$ dos espécimes $\left(\mathrm{CL}_{50}\right)$.

\section{RESULTADOS E DISCUSSÃO}

Da consulta realizada, foram obtidos 239 artigos, sendo 21 da base de dados Scielo, 161 da Science Direct e 57 da Scopus.

A partir da exclusão de 16 artigos (5 que apareciam em mais de uma base de dados e 11 cujo conteúdo não estava disponível na íntegra), 223 artigos foram analisados quanto aos demais critérios de elegibilidade. Nesse sentido, 112 artigos foram excluídos dessa etapa de triagem por contemplarem outras espécies de culicídeos (63) e pela não realização de ensaio larvicida (49). Dos 111 artigos selecionados após essa triagem, 42 não apresentavam valores referentes à $\mathrm{CL}_{50}$ dos extratos vegetais e/ou óleos essenciais utilizados no desafio sobre larvas de Ae. aegypti e Ae. albopictus, o que resultou na análise, ao final da seleção, de 69 artigos, conforme ilustra a figura 1.

Figura 1. Fluxograma representativo do processo de busca e seleção dos artigos.
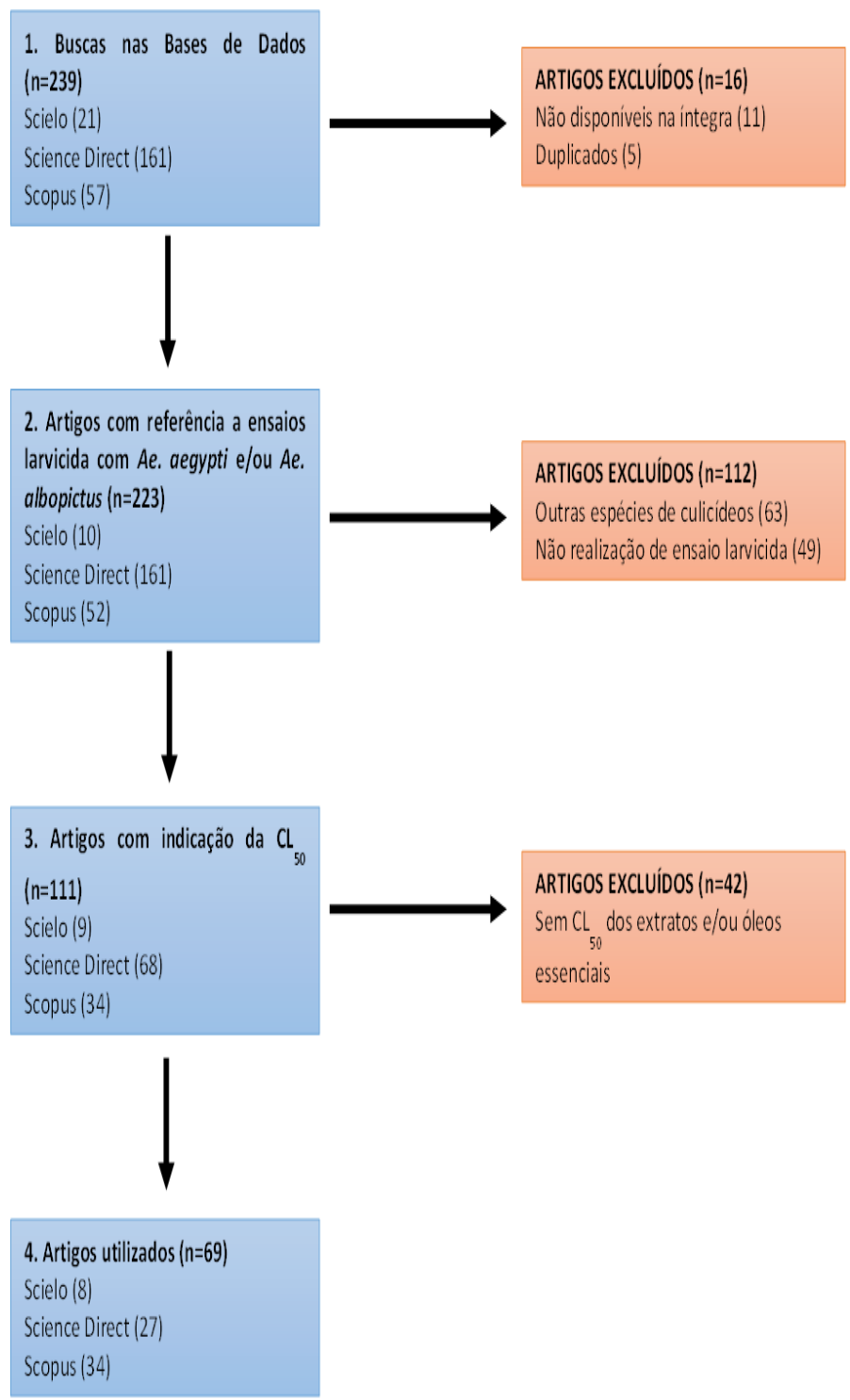

A análise dos artigos selecionados mostrou a utilização de 219 espécies vegetais, pertencentes a 43 famílias distintas, com destaque para Lamiaceae (25 espécies), Myrtaceae (22 espécies), Rutaceae (20 espécies), Cupressaceae (16 espécies) Asteraceae (15 espécies), Pinaceae (11 espécies), Apiceae (10 
espécies) e Lauraceae (10 espécies).

Em relação ao Ae. aegypti, foram testados 158 óleos essenciais, dos quais 141 (89,2\%) exibiram um significativo efeito larvicida, tendo, para isso, como parâmetro, valores de $\mathrm{CL}_{50}$ iguais ou inferiores a 100 ppm $^{10}$. Já em relação aos extratos testados (67), 15 (22,3\%) tiveram os valores da $\mathrm{CL}_{50}$ enquadrados no parâmetro acima mencionado (Tabela 1).

Tabela 1. Extratos e óleos essenciais obtidos de distintas partes vegetais e seu efeito larvicida sobre Aedes aegypti, 2000 a 2016.

\begin{tabular}{|c|c|c|c|c|}
\hline Família/Espécie & Estrutura vegetal & Produto obtido & CL50 (ppm) & Referência \\
\hline \multicolumn{5}{|l|}{ ACANTHACEAE } \\
\hline \multirow[t]{6}{*}{ Andrographis paniculata } & & Extrato benzênico & 119,5 & Govindarajan, $2011^{17}$ \\
\hline & & Extrato hexânico & 146,3 & \\
\hline & & Extrato acetato etílico & 124,2 & \\
\hline & Folha & Extrato metanólico & 110,1 & \\
\hline & & Extrato clorofórmico & 99,5 & \\
\hline & & $\begin{array}{l}\text { Extrato de éter de } \\
\text { petróleo }\end{array}$ & 143,2 & Renugadevi et al., $2013^{18}$ \\
\hline \multirow[t]{3}{*}{ Andrographis lineata nees } & & Extrato aquoso & 129,3 & \\
\hline & & $\begin{array}{l}\text { Extrato de éter de } \\
\text { petróleo }\end{array}$ & 193,1 & \\
\hline & & Extrato aquoso & 152,2 & \\
\hline
\end{tabular}

\section{ACORACEAE}

\begin{tabular}{|c|c|c|c|c|}
\hline Acorus calamus & Raiz & Óleo essencial & 99,4 & Dias \& Moraes, $2014^{19}$ \\
\hline \multicolumn{5}{|l|}{ AMARANTHACEAE } \\
\hline Chenopodium ambrosioides & Folha & Óleo essencial & 9,1 & Dias \& Moraes, $2014^{19}$ \\
\hline \multicolumn{5}{|l|}{ ANACARDIACEAE } \\
\hline Anacardium humile & Folha & Óleo essencial & 20,9 & Dias \& Moraes, $2014^{19}$ \\
\hline Schinus molle & & & 9,6 & \\
\hline Spondias purpurea & & & 39,7 & \\
\hline Melanochyla fasciculiflora & Folha e Caule & Extrato metanólico & $619,2-753$ & Yousaf \& Zuharah, $2015^{20}$ \\
\hline Gluta rengas & & & $418,8-634$ & \\
\hline Anacardium occidentale & & & $656,4-804,2$ & \\
\hline Mangifera indica & & & $523,9-697,3$ & \\
\hline \multicolumn{5}{|l|}{ ANNONACEAE } \\
\hline Guatteria blepharophylla & Folha & Óleo essencial & 58,7 & Aciole et al., $2011^{21}$ \\
\hline Guatteria friesiana & & & 52,6 & \\
\hline Guatteria hispida & & & 85,7 & \\
\hline Rollinia leptopetala & Caule & & 34,7 & Dias \& Moraes, $2014^{19}$ \\
\hline \multicolumn{5}{|l|}{ APIACEAE } \\
\hline \multirow[t]{2}{*}{ Anethum graveolens } & Folha & Óleo essencial & & \\
\hline & & & & Dias \& Moraes, $2014^{19}$ \\
\hline Apium graveolens & Semente & & 16,1 & Kumar et al., 2014²2 \\
\hline Carum carvi & & & 54,6 & Dias \& Moraes, $2014^{19}$ \\
\hline Foeniculum vulgare & Fruto & & 49,3 & \\
\hline $\begin{array}{l}\text { Heracleum pastinacifolium } \\
\text { incanum }\end{array}$ & & & 71,8 & \\
\hline $\begin{array}{l}\text { Heracleum pastinacifolium } \\
\text { transcaucasicum }\end{array}$ & & & 69,7 & \\
\hline Heracleum sprengelianum & Folha & & 37,5 & Govindarajan \& Benelli, $2016^{23}$ \\
\hline Pimpinella anisum & Semente & & $<100$ & Dias \& Moraes, $2014^{19}$ \\
\hline
\end{tabular}


452 Produtos vegetais no controle de Aedes aegypti e Aedes albopictus

\begin{tabular}{|c|c|c|c|c|}
\hline Família/Espécie & Estrutura vegetal & Produto obtido & CL50 (ppm) & Referência \\
\hline Seseli diffusum & & & 126,13 & Kabir et al., $2013^{24}$ \\
\hline \multicolumn{5}{|l|}{ APOCYNACEAE } \\
\hline Tabernaemontana cymosa & Semente & Extrato etanólico & 35,1 & Castillo et al., $2012^{25}$ \\
\hline \multicolumn{5}{|l|}{ ARALIACEAE } \\
\hline Dendropanax morbifera & Flor & Óleo essencial & 35,1 & Castillo et al., $2012^{25}$ \\
\hline \multicolumn{5}{|l|}{ ARECACEAE } \\
\hline Cocos nucifera & Fruto & Óleo essencial & 294,9 & Fazal et al., $2013^{26}$ \\
\hline \multicolumn{5}{|l|}{ ARISTOLOCHIACEAE } \\
\hline Asarum heterotropoides & Raiz & Óleo essencial & 23,8 & Dias \& Moraes, $2014^{19}$ \\
\hline \multicolumn{5}{|l|}{ ASTERACEAE } \\
\hline Ageratum conyzoides & Folha & Óleo essencial & 61,5 & Furtado et al., $2005^{27}$ \\
\hline Artemisia abrotamum & & & 620 & Leyva et al., $2008^{28}$ \\
\hline Baccharis sp. & & & 14,7 & Dias \& Moraes, $2014^{19}$ \\
\hline Coreopsis fasciculata & & & 26,8 & \\
\hline Senecio adenophylloides & & & 43,4 & \\
\hline \multirow[t]{4}{*}{ Sphaeranthus indicus } & & Extrato hexânico & 966 & Tennyson et al., $2012^{29}$ \\
\hline & & Extrato de éter dietílico & 869,3 & \\
\hline & & Extrato de diclorometano & 1559,5 & \\
\hline & & Extrato acetato etílico & 201,1 & \\
\hline Tagetes erecta & & Óleo essencial & 15,3 & Dias \& Moraes, $2014^{19}$ \\
\hline Tagetes filifolia & & & 47,7 & \\
\hline Tagetes minuta & & & 52,3 & Lima et al., $2009^{30}$ \\
\hline Tagetes patula & & & 13,5 & Dharmagadda et al., $2005^{31}$ \\
\hline Tagetes pusilla & & & 14,1 & Dias \& Moraes, $2014^{19}$ \\
\hline Tridax procumbens & & $\begin{array}{l}\text { Extrato de éter de } \\
\text { Petróleo }\end{array}$ & 219 & $\begin{array}{l}\text { Rajasekaran \& Duraikannan, } \\
2012^{32}\end{array}$ \\
\hline Vanillosmopsis arborea & Caule & Óleo essencial & 15,9 & Furtado et al., $2005^{27}$ \\
\hline \multicolumn{5}{|l|}{ BORAGINACEAE } \\
\hline Auxemma glazioviana & Caule & Óleo essencial & 2,5 & Dias \& Moraes, $2014^{19}$ \\
\hline Cordia curassavica & Folha & & 87,7 & \\
\hline Cordia leucomalloides & & & 63,1 & \\
\hline \multicolumn{5}{|l|}{ BURSERACEAE } \\
\hline Boswellia carteri & Caule & Óleo essencial & 10 & Dias \& Moraes, $2014^{19}$ \\
\hline \multicolumn{5}{|l|}{ CAESALPINIACEAE } \\
\hline Copaifera reticulata & Caule & Óleo essencial & 8,9 & Silva et al., $2007^{33}$ \\
\hline \multicolumn{5}{|l|}{ CARICACEAE } \\
\hline Carica papaya & Folha e Semente & Extrato etanólico & 107 & Wahyuni, $2015^{34}$ \\
\hline \multicolumn{5}{|l|}{ CONVOLVULACEAE } \\
\hline Ipomoea cairica & Folha, Flor e Raiz & Extrato metanólico & $12,2-37,5$ & Ishak et al., $2014^{35}$ \\
\hline \multicolumn{5}{|l|}{ CUCURBITACEAE } \\
\hline \multirow[t]{4}{*}{ Citrullus colocynthis } & Folha & Extrato hexânico & 1087,6 & Tennyson et al., $2012^{29}$ \\
\hline & & Extrato de éter dietílico & 1022,3 & \\
\hline & & Extrato de diclorometano & 515,6 & \\
\hline & & Extrato acetato etílico & 1212,9 & \\
\hline \multicolumn{5}{|l|}{ CUPRESSACEAE } \\
\hline Calocedrus formosana & Caule & Óleo essencial & 51,8 & Cheng et al., $2003^{10}$ \\
\hline Callitris glaucophylla & & & 0,6 & Dias \& Moraes, $2014^{19}$ \\
\hline
\end{tabular}




\begin{tabular}{|c|c|c|c|c|}
\hline Família/Espécie & Estrutura vegetal & Produto obtido & CL50 (ppm) & Referência \\
\hline Chamaecyparis formosensis & & & 38,6 & \\
\hline Cryptomeria japonica & Folha & & 28,4 & \\
\hline Cunninghamia konishii & Caule & & 85,7 & \\
\hline Juniperus macropoda & Fruto & & $<100$ & \\
\hline Juniperus virginiana & Caule & & 1 & \\
\hline Taiwania cryptomerioides & & & 79,8 & \\
\hline \multicolumn{5}{|l|}{ CYMODOCEACEAE } \\
\hline \multirow[t]{2}{*}{ Syringodium isoetifoliu } & Raízes & Extrato etanólico & 0,06 & Ali et al., $2012^{36}$ \\
\hline & Folha & & 0,06 & \\
\hline Cymodocea serrulata & & & 0,07 & \\
\hline \multicolumn{5}{|l|}{ EUPHORBIACEAE } \\
\hline \multirow[t]{4}{*}{ Cleistanthus collinus } & Folha & Extrato hexânico & 1291,2 & Tennyson et al., $2012^{29}$ \\
\hline & & Extrato de éter dietílico & 837,3 & \\
\hline & & Extrato de diclorometano & 755,2 & \\
\hline & & Extrato acetato etílico & 560,4 & \\
\hline Croton argyrophylloides & & Óleo essencial & 50 & Lima et al., $2006^{37}$ \\
\hline Croton heliotropiifolius & & & 544 & Dória et al., $2010^{38}$ \\
\hline Croton nepetaefolius & & & 66,4 & Lima et al., $2006^{37}$ \\
\hline Croton pulegiodorus & & & 159 & Dória et al., $2010^{38}$ \\
\hline Croton regelianus & & & 24,2 & Dias \& Moraes, $2014^{19}$ \\
\hline Croton sonderianus & & & 54,5 & Lima et al., $2006^{37}$ \\
\hline Croton zehntneri & & & 26,2 & \\
\hline \multicolumn{5}{|l|}{ FABACEAE } \\
\hline Bauhinia acuruana & Folha & Óleo essencial & 56,2 & Dias \& Moraes, $2014^{19}$ \\
\hline \multirow[t]{2}{*}{ Copaifera multijuga } & Caule e Folha & Extrato etanólico & $81-166$ & Trindade et al., $2013^{39}$ \\
\hline & & Óleo essencial & 18 & \\
\hline \multirow[t]{4}{*}{ Dalbergia oliveri } & Folha & Extrato hexânico & 153,7 & Pluempanupat et al., $2013^{40}$ \\
\hline & & Extrato de diclorometano & 169,9 & \\
\hline & & Extrato de acetato etílico & 163,7 & \\
\hline & & Extrato metanólico & 255,6 & \\
\hline Hymenaea courbaril & Fruto & Óleo essencial & 14.8 & Dias \& Moraes, $2014^{19}$ \\
\hline \multirow[t]{2}{*}{ Pongamia pinnata } & Caule & Extrato metanólico & 118,2 & Kolli et al., $2013^{41}$ \\
\hline & & Extrato hidroalcoólico & 128,3 & \\
\hline \multicolumn{5}{|l|}{ GUTTIFERAE } \\
\hline Hypericum polyanthemum & Folha & Extrato de acetona & 121 & Silva et al., $2013^{42}$ \\
\hline \multicolumn{5}{|l|}{ LAMIACEAE } \\
\hline Hyptis martiusii & Folha & Óleo essencial & 18,5 & Dias \& Moraes, $2014^{19}$ \\
\hline \multirow[t]{4}{*}{ Hyptis suaveolens } & & Extrato hexânico & 543,6 & Tennyson et al., $2012^{29}$ \\
\hline & & Extrato de éter dietílico & 1443,5 & \\
\hline & & Extrato de diclorometano & 1292,3 & \\
\hline & & Extrato acetato etílico & 853 & \\
\hline Lavandula gibsoni & & Óleo essencial & 48,3 & Dias \& Moraes, $2014^{19}$ \\
\hline \multirow[t]{4}{*}{ Leucas aspera } & & Extrato hexânico & 1359,2 & Tennyson et al., $2012^{29}$ \\
\hline & & Extrato de éter dietílico & 927,6 & \\
\hline & & Extrato de diclorometano & 844,6 & \\
\hline & & Extrato acetato etílico & 483,2 & \\
\hline
\end{tabular}


454 Produtos vegetais no controle de Aedes aegypti e Aedes albopictus

\begin{tabular}{|c|c|c|c|c|}
\hline Família/Espécie & Estrutura vegetal & Produto obtido & CL50 (ppm) & Referência \\
\hline \multirow[t]{2}{*}{ Mentha piperita } & & Óleo essencial & 47,5 & Kalaivani et al., $2012^{43}$ \\
\hline & & & 111,9 & Kumar et al., $2011^{44}$ \\
\hline Mentha spicata & & & 56 & Dias \& Moraes, $2014^{19}$ \\
\hline Mentha villosa & & & 45 & Lima et al., $2014^{45}$ \\
\hline Ocimum americanum & & & 67 & Dias \& Moraes, $2014^{19}$ \\
\hline \multirow[t]{2}{*}{ Ocimum basilicum } & Caule & & 148,5 & Kalaivani et al., $2012^{43}$ \\
\hline & Folha & & 66,9 & Furtado et al., $2005^{27}$ \\
\hline Ocimum gratissimum & & & 104,5 & \\
\hline Ocimum lamiifolium & & & 8,6 & Dias \& Moraes, $2014^{19}$ \\
\hline Ocimum sanctum & & & 29,7 & \\
\hline Ocimum suave & & & 29,8 & \\
\hline Ocimum tenuiflorum & & & 71,2 & Furtado et al., $2005^{27}$ \\
\hline Origanum scabrum & & & 67,1 & Govindarajan et al., $2016^{46}$ \\
\hline Plectranthus amboinicus & & & 51,8 & Dias \& Moraes, $2014^{19}$ \\
\hline Plectranthus mollis & & & 25,3 & \\
\hline Thymus vulgaris & & & 17,3 & \\
\hline
\end{tabular}

\begin{tabular}{|c|c|c|c|c|}
\hline \multicolumn{5}{|l|}{ LAURACEAE } \\
\hline Cinnamomum impressicostatum & Folha & Óleo essencial & 10,7 & Dias \& Moraes, $2014^{19}$ \\
\hline Cinnamomum microphyllum & & & 6,7 & \\
\hline Cinnamomum mollissimum & & & 10,2 & \\
\hline Cinnamomum osmophloeum & & & 120 & Cheng et al., $2003^{10}$ \\
\hline Cinnamomum pubescens & & & 12,8 & Dias \& Moraes, $2014^{19}$ \\
\hline Cinnamomum rhyncophyllum & & & 6 & \\
\hline Cinnamomum scortechinii & & & 21,5 & \\
\hline Cinnamomum sintoc & & & 41 & \\
\hline Cinnamomum zeylanicum & & & 79,7 & \\
\hline \multirow[t]{2}{*}{ Persea americana } & Semente & Extrato etanólico & 16,4 & Torres et al., $2014^{47}$ \\
\hline & & Extrato hexânico & 9,8 & \\
\hline \multicolumn{5}{|l|}{ MALVACEAE } \\
\hline \multirow[t]{4}{*}{ Abutilon indicum } & Folha & Extrato hexânico & 261,3 & Tennyson et al., $2012^{29}$ \\
\hline & & Extrato de éter dietílico & 1442,3 & \\
\hline & & Extrato de diclorometano & 1434,5 & \\
\hline & & Extrato acetato etílico & 898,8 & \\
\hline Sida acuta & & Extrato metanólico & 42 & Govindarajan, $2010^{48}$ \\
\hline \multicolumn{5}{|l|}{ MELIACEAE } \\
\hline Azadirachta indica & Semente & Óleo essencial & 117,1 & Fazal et al., $2013^{26}$ \\
\hline Guarea humaitensis & Folha & & 48,6 & Dias \& Moraes, $2014^{19}$ \\
\hline Guarea scabra & & & 98,6 & \\
\hline Trichilia hirta & Semente & Extrato etanólico & 219,2 & Castillo et al., $2012^{25}$ \\
\hline \multicolumn{5}{|l|}{ MYRTACEAE } \\
\hline \multirow[t]{2}{*}{ Eucalyptus camaldulensis } & Folha & Óleo essencial & $31-55,3$ & Cheng et al., $2009^{13}$ \\
\hline & Semente & & 26,7 & Lucia et al., $2008^{49}$ \\
\hline Eucalyptus citriodora & Folha & & 68,9 & Fazal et al., $2013^{26}$ \\
\hline Eucalyptus dunnii & Semente & & 25,2 & Lucia et al., $2008^{49}$ \\
\hline Eucalyptus globulus & Folha & & 52,9 & Dias \& Moraes, $2014^{19}$ \\
\hline Eucalyptus grandis & & & 32,4 & \\
\hline
\end{tabular}




\begin{tabular}{|c|c|c|c|c|}
\hline Família/Espécie & Estrutura vegetal & Produto obtido & CL50 (ppm) & Referência \\
\hline Eucalyptus gunnii & Semente & & 21,1 & Lucia et al., $2008^{49}$ \\
\hline Eucalyptus saligna & Folha & & 22,1 & \\
\hline Eucalyptus tereticornis & & & 22,1 & \\
\hline Eucalyptus urophylla & & & 95,5 & Dias \& Moraes, $2014^{19}$ \\
\hline Eugenia melanadenia & & & 85 & \\
\hline Eugenia piauhiensis & & & 230 & Dias et al., $2015^{50}$ \\
\hline Eugenia triquetra & & & 64,8 & Dias \& Moraes, 201419 \\
\hline Melaleuca leucadendron & & & 120 & Leyva et al., $2008^{28}$ \\
\hline Myrtus communis & & & 50 & Dias \& Moraes, $2014^{19}$ \\
\hline Pimenta dioica & & & 38,8 & \\
\hline Pimenta pseudocaryophyllus & & & 44,1 & \\
\hline Pimenta racemosa & & & 27 & \\
\hline Psidium guajava & & & 24,7 & \\
\hline Psidium myrsinites & & & 292 & Dias et al., $2015^{50}$ \\
\hline Psidium rotundatum & & & 63 & Dias \& Moraes, $2014^{19}$ \\
\hline Syzygium aromaticum & & & 92,5 & Fayemiwo et al., $2014^{51}$ \\
\hline \multicolumn{5}{|l|}{ PINACEAE } \\
\hline Pinus caribaea & Folha & Óleo essencial & 51 & Dias \& Moraes, $2014^{19}$ \\
\hline Pinus kesiya & & & 57 & Govindarajan et al., $2016^{52}$ \\
\hline Pinus sylvestris & & & 128,1 & Fayemiwo et al., $2014^{51}$ \\
\hline Pinus tropicalis & & & 42 & Dias \& Moraes, $2014^{19}$ \\
\hline \multicolumn{5}{|l|}{ PIPERACEAE } \\
\hline \multirow[t]{2}{*}{ Piper aduncum } & Folha & Óleo essencial & 46 & Santana et al., $2015^{53}$ \\
\hline & Fruto & & 30,2 & Dias \& Moraes, $2014^{19}$ \\
\hline Piper arboreum & Folha & & 55 & Santana et al., $2015^{53}$ \\
\hline Piper auritum & & & 17 & Dias \& Moraes, $2014^{19}$ \\
\hline Piper hostmanianum & & & 54 & \\
\hline Piper klotzschianum & Semente & & 13,2 & \\
\hline \multirow[t]{2}{*}{ Piper marginatum } & Folha & & 34 & Santana et al., $2015^{53}$ \\
\hline & Caule & & 19,9 & Dias \& Moraes, $2014^{19}$ \\
\hline Piper nigrum & Semente & & 9,1 & \\
\hline \multirow[t]{3}{*}{ Piper ovatum } & Raiz & Extrato etanólico & 2,9 & Kabir et al., $2013^{24}$ \\
\hline & Caule & & 28 & \\
\hline & Folha & & 92 & \\
\hline Piper permucronatum & & Óleo essencial & 36 & Dias \& Moraes, $2014^{19}$ \\
\hline \multicolumn{5}{|l|}{ POACEAE } \\
\hline Cymbopogon citratus & Folha & Óleo essencial & 63,8 & Furtado et al., $2005^{27}$ \\
\hline Cymbopogon winterianus & & & 54,6 & \\
\hline Vetiveria zizanioides & Raiz & & 31,5 & Dias \& Moraes, $2014^{19}$ \\
\hline \multicolumn{5}{|l|}{ RANUCULACEAE } \\
\hline \multirow[t]{2}{*}{ Nigella sativa } & Folha & Óleo essencial & 32,1 & Dias \& Moraes, $2014^{19}$ \\
\hline & Semente & & 99,9 & Raj et al., $2015^{54}$ \\
\hline \multicolumn{5}{|l|}{ RUTACEAE } \\
\hline Amyris balsamifera & Caule & Óleo essencial & 1 & Dias \& Moraes, $2014^{19}$ \\
\hline
\end{tabular}




\begin{tabular}{|c|c|c|c|c|}
\hline Família/Espécie & Estrutura vegetal & Produto obtido & CL50 (ppm) & Referência \\
\hline Chloroxylon swietenia & Folha & & 16,5 & \\
\hline Citrus limon & Fruto & & 95,8 & Furtado et al., $2005^{27}$ \\
\hline Citrus sinensis & Folha & Extrato hexânico & 446,4 & Warikoo et al., $2012^{55}$ \\
\hline Clausena anisata & & Óleo essencial & 130,1 & Govindarajan, $2010^{48}$ \\
\hline \multirow[t]{2}{*}{ Clausena dentata } & & Óleo essencial & 140,2 & Rajkumar \& Jebanesan, $2010^{56}$ \\
\hline & & $\begin{array}{l}\text { Extratos metanólico, } \\
\text { clorofórmico, benzênico e } \\
\text { de acetona }\end{array}$ & $0,16-1,2$ & Manjari et al., $2014^{57}$ \\
\hline Clausena excavata & & Óleo essencial & 37,1 & Dias \& Moraes, $2014^{19}$ \\
\hline Feronia limonia & & & 11,5 & \\
\hline Murraya exotica & & & 74,7 & Krishnamoorthy et al., $2015^{58}$ \\
\hline \multirow[t]{4}{*}{ Murraya koenigii } & & Extrato hexânico & 856,7 & Tennyson et al., $2012^{29}$ \\
\hline & & Extrato de éter dietílico & 511,1 & \\
\hline & & Extrato de diclorometano & 1452,5 & \\
\hline & & Extrato acetato etílico & 1421 & \\
\hline Ruta graveolens & & Óleo essencial & 21,2 & Dias \& Moraes, $2014^{19}$ \\
\hline Zanthoxylum armatum & Semente & & 54 & \\
\hline Zanthoxylum articulatum & Folha & & 77,6 & \\
\hline Zanthoxylum limonella & Fruto & & 24,6 & Pitasawat et al., $2007^{59}$ \\
\hline Zanthoxylum oxyphyllum & Folha & & 7,5 & Dias \& Moraes, $2014^{19}$ \\
\hline \multicolumn{5}{|l|}{ SANTALACEAE } \\
\hline Santalum album & Caule & Óleo essencial & 10 & Dias \& Moraes, $2014^{19}$ \\
\hline \multicolumn{5}{|l|}{ SOLANACEAE } \\
\hline Datura stramonium & Folha & $\begin{array}{l}\text { Extrato de éter de } \\
\text { petróleo }\end{array}$ & 288 & $\begin{array}{l}\text { Rajasekaran \& Duraikannan, } \\
2012^{32}\end{array}$ \\
\hline \multicolumn{5}{|l|}{ SCROPHULAREACEAE } \\
\hline Capraria biflora & Folha & Óleo essencial & 73,3 & Dias \& Moraes, $2014^{19}$ \\
\hline Stemodia maritima & Caule & & 22,9 & \\
\hline \multicolumn{5}{|l|}{ TAXODIACEAE } \\
\hline Cunninghamia lanceolata & Caule & Óleo essencial & 106,4 & Cheng et al., $2003^{10}$ \\
\hline Taiwania cryptomerioides & & & 79,8 & \\
\hline \multicolumn{5}{|l|}{ UMBELLIFERACEAE } \\
\hline Petroselinum crispum & Fruto & Óleo essencial & 43,2 & Intirach et al., $2016^{60}$ \\
\hline \multicolumn{5}{|l|}{ VERBENACEAE } \\
\hline Lantana camara & Folha & Óleo essencial & 42,3 & $\begin{array}{l}\text { Rajasekaran \& Duraikannan, } \\
2012^{32}\end{array}$ \\
\hline Lippia adoensis & & & 47,1 & Dias \& Moraes, $2014^{19}$ \\
\hline Lippia gracilis & & & 282 & Dias et al., $2015^{50}$ \\
\hline Lippia sidoides & & & 45,4 & Furtado et al., $2005^{27}$ \\
\hline \multicolumn{5}{|l|}{ ZINGIBERACEAE } \\
\hline Alpinia purpurata & Flor & Óleo essencial & 80,7 & Dias \& Moraes, $2014^{19}$ \\
\hline Curcuma aromatica & Caule & & 36,3 & \\
\hline Curcuma domestica & & & 20,9 & \\
\hline Curcuma longa & & & 115,6 & Kalaivani et al., $2012^{43}$ \\
\hline Curcuma xanthorrhiza & & & 74,2 & Dias \& Moraes, $2014^{19}$ \\
\hline Curcuma zedoaria & & & 31,8 & Pitasawat et al., $2007^{59}$ \\
\hline Etlingera elatior & Flor & & 26,6 & Dias \& Moraes, $2014^{19}$ \\
\hline
\end{tabular}




\begin{tabular}{|c|c|c|c|c|}
\hline Família/Espécie & Estrutura vegetal & Produto obtido & CL50 (ppm) & Referência \\
\hline Zingiber nimmonii & Caule & & 44,4 & Govindarajan et al., $2016^{61}$ \\
\hline Zingiber officinale & & & 40,5 & Kalaivani et al., $2012^{43}$ \\
\hline \multicolumn{5}{|l|}{ ZYGOPHYLACEAE } \\
\hline Peganum harmala & Folha & Óleo essencia & 60,9 & Fazal et al., $2013^{26}$ \\
\hline \multirow[t]{3}{*}{ Tribulus terrestris } & & Extrato etanólico & 376,4 & El-Sheikh et al., $2016^{62}$ \\
\hline & & Extrato de acetona & 173,2 & \\
\hline & & $\begin{array}{l}\text { Extrato de éter de } \\
\text { petróleo }\end{array}$ & 64,6 & \\
\hline
\end{tabular}

No tocante ao Ae. albopictus, 43 óleos essenciais tiveram sua ação larvicida testada, dos quais 32 (74,4\%) com valores de $\mathrm{CL}_{50}$ iguais ou menores a $100 \mathrm{ppm}$, ao passo que, dos 18 extratos testados, 6 (33,3\%) exibiram valores de $\mathrm{CL}_{50}$ dentro dos parâmetros acima considerados (Tabela 2).

Tabela 2. Extratos e óleos essenciais obtidos de distintas partes vegetais e seu efeito larvicida sobre Aedes albopictus, 2000 a 2016.

\begin{tabular}{|c|c|c|c|c|}
\hline Família/Espécie & Estrutura vegetal & Produto obtido & CL50 (ppm) & Referência \\
\hline \multicolumn{5}{|l|}{ AMARYLLIDACEAE } \\
\hline Allium macrostemon & Caule & Óleo essencial & 72,8 & Liu et al., $2014^{63}$ \\
\hline \multicolumn{5}{|l|}{ ANACARDIACEAE } \\
\hline $\begin{array}{l}\text { Melanochyla fasciculiflora } \\
\text { Gluta rengas } \\
\text { Anacardium occidentale } \\
\text { Mangifera indica }\end{array}$ & Folha e Caule & Extrato metanólico & $\begin{array}{l}474,7-727,6 \\
240,1-589,6 \\
638,4-791,1 \\
431,1-722,2\end{array}$ & Yousaf \& Zuharah, $2015^{20}$ \\
\hline \multicolumn{5}{|l|}{ APIACEAE } \\
\hline Coriandrum sativum & Fruto & Óleo essencial & 421 & Benelli et al., $2013^{64}$ \\
\hline \multicolumn{5}{|l|}{ ASTERACEAE } \\
\hline $\begin{array}{l}\text { Achillea millefolium } \\
\text { Helichrysum italicum }\end{array}$ & Folha & Óleo essencial & $\begin{array}{l}211,3 \\
178,1\end{array}$ & Conti et al., $2010^{65}$ \\
\hline \multicolumn{5}{|l|}{ CANNABACEAE } \\
\hline $\begin{array}{l}\text { Cannabis sativa } \\
\text { Humulus lupulus }\end{array}$ & Folha & Óleo essencial & $\begin{array}{l}0,3 \\
0,3\end{array}$ & Bedini et al., $2016^{66}$ \\
\hline \multicolumn{5}{|l|}{ CONVOLVULACEAE } \\
\hline Ipomoea cairica & $\begin{array}{l}\text { Folha, Flor e } \\
\text { Raízes }\end{array}$ & Extrato metanólico & $21,7-37,6$ & Ishak et al., $2014^{35}$ \\
\hline \multicolumn{5}{|l|}{ COSTACEAE } \\
\hline Saussurea lappa & Raiz & Óleo essencial & 12,4 & Liu et al., $2012^{67}$ \\
\hline \multicolumn{5}{|l|}{ CUPRESSACEAE } \\
\hline $\begin{array}{l}\text { Chamaecyparis lawsoniana } \\
\text { Cupressus arizonica glabra } \\
\text { Cupressus benthamii } \\
\text { Cupressus macrocarpa } \\
\text { Cupressus sempervirens } \\
\text { Cupressus torulosa } \\
\text { Juniperus phoenicea } \\
\text { Tetraclinis articulata }\end{array}$ & Folha & Óleo essencial & $\begin{array}{l}47,9 \\
64,8 \\
37,5 \\
54,6 \\
54,7 \\
57,1\end{array}$ & Giatropoulos et al., $2013^{68}$ \\
\hline \multicolumn{5}{|l|}{ EUPHORBIACEAE } \\
\hline Ricinus communis & Semente & Extrato de acetona & 11,6 & Mandal, $2010^{69}$ \\
\hline \multicolumn{5}{|l|}{ FABACEAE } \\
\hline Tephrosia vogelii & Folha & Extrato aquoso & 1,1 & Li et al., $2015^{70}$ \\
\hline
\end{tabular}




\begin{tabular}{|c|c|c|c|c|}
\hline Família/Espécie & Estrutura vegetal & Produto obtido & CL50 (ppm) & Referência \\
\hline \multicolumn{5}{|l|}{ LAMIACEAE } \\
\hline $\begin{array}{l}\text { Coleus aromaticus } \\
\text { Hyptis suaveolens } \\
\text { Lavandula angustifolia } \\
\text { Ocimum basilicum. } \\
\text { Ocimum gratissimum } \\
\text { Rosmarinus officinalis } \\
\text { Salvia dorisiana } \\
\text { Salvia elegans } \\
\text { Salvia splendens Blue Ribbon } \\
\text { Salvia splendens Scarlet Sage }\end{array}$ & Folha & Óleo essencial & $\begin{array}{r}67,9 \\
240,3 \\
>250 \\
11,9 \\
26,1 \\
>250 \\
76,7 \\
46,4 \\
59,2 \\
92,7\end{array}$ & 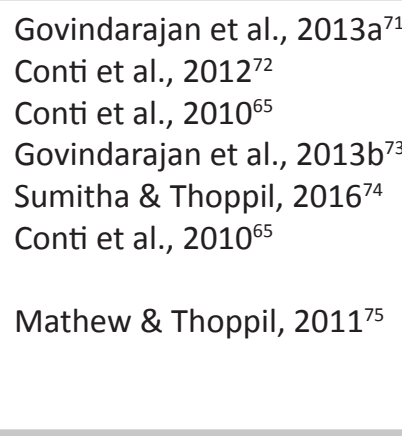 \\
\hline \multicolumn{5}{|l|}{ LAURACEAE } \\
\hline Cinnamomum osmophloeum & Folha & Óleo essencial & 40,8 & Cheng et al., $2009^{76}$ \\
\hline \multicolumn{5}{|l|}{ MALVACEAE } \\
\hline Abutilon indicum & Folha & $\begin{array}{l}\text { Extrato hexânico } \\
\text { Extrato de éter } \\
\text { dietílico } \\
\text { Extrato de } \\
\text { diclorometano } \\
\text { Extrato acetato } \\
\text { etílico }\end{array}$ & $\begin{array}{r}261,3 \\
1442,3 \\
1434,5 \\
898,8\end{array}$ & $\begin{array}{l}\text { Tennyson et al., } 2012^{29} \\
\text { Govindarajan, } 2010^{48}\end{array}$ \\
\hline Sida acuta & & Extrato metanólico & 42,0 & \\
\hline
\end{tabular}

\section{MYRTACEAE}

Eucalyptus camaldulensis

Folha

Semente

Myrtus communis

Folha

Raiz

Syzygium guineense

\section{PINACEAE}

Pinus canariensis

Pinus halepensis

Pinus nigra

Pinus pinaster

Pinus stankewiczii

Pinus strobus

\section{$31,0-55,3$ Cheng et al., $2009^{13}$}

Óleo essencial

26,7 Lucia et al., $2008^{49}$

50 Dias \& Moraes, $2014^{19}$

$>250$ Conti et al., $2010^{65}$

66,8 Francine et al., 2016 77
Pinus brutia

67

$>200$

70,2

Caule Óleo essencial

152,6 Koutsaviti et al., $2015^{78}$

$>200$

81,6

127,9

\section{RUTACEAE}

Citrus limon

Óleo essencial

95,8 Furtado et al., 2005 27

25,0 Giatropoulos et al., $2012^{79}$

Citrus paradisi

Fruto

37.03

28,6

Citrus sinensis

Folha

Extrato hexânico

Monodora myristica

Fruto

Extrato etanólico

Ruta chalepensis

Óleo essencial

Toddalia asiatica

Zanthoxylum heitzii
Raiz

Extrato etanólico
446,4 Warikoo et al., 201255

110,9 Francine et al., 201677

35,6 Conti et al., 201380

69,0 Liu et al., $2013^{81}$

229 Francine et al., 2016

288

47,9 
Observa-se que a maior parte dos estudos realizados utilizou o Ae. aegypti como a espécie vetora mais desafiada nos ensaios larvicida com extratos vegetais e óleos essenciais (Tabelas 1 e 2). Isso se deve, certamente, ao fato de que este seja o vetor mais amplamente disseminado nos grandes centros urbanos, relacionado com a transmissão dos vírus Dengue, Chikungunya e Zika, embora estudos tenham demonstrado a gradativa domiciliação do Ae. albopictus, chegando, inclusive, a ter suas formas imaturas coexistindo nos mesmos criadouros que as do Ae. aegypti no intradomicílio dos imóveis pesquisados ${ }^{11}$.

Os óleos essenciais apresentam-se como misturas complexas de substâncias voláteis, lipofílicas, de baixo peso molecular e geralmente odoríferas, tendo como constituintes majoritários, na maioria das vezes, moléculas de natureza terpênica ${ }^{12}$. Por constituírem, muitas vezes, a fração ativa dos extratos vegetais, são importantes fontes naturais de moléculas bioativas, o que os torna os mais eficientes inseticidas vegetais ${ }^{13}$. Essa característica pode ser corroborada, entre as famílias com mais espécies estudadas, pelos valores das $\mathrm{CL}_{50}$ obtidos a partir dos óleos essenciais de Tagetes patula (Asteraceae; 13,5 ppm), Apium graveolens (Apiace; 16,1 ppm), Callitris glaucophyla (Cupressaceae; 0,6 ppm), Ocimum lamiifolium (Lamiaceae; 8,6 ppm), Cinnamomum rhyncophyllum (Lauraceae; 6 ppm), Eucalyptus gunii (Myrtaceae; 21,1 ppm), Pinus tropicalis (Pinaceae; 42 ppm) e Amyris balsamifera (Rutaceae; 1 ppm) (Tabelas 1 e 2).

As plantas produzem uma quantidade imensurável de compostos bioativos, classificados como metabólitos secundários, os quais são dotados de estruturas químicas de altíssima complexidade e com um espectro de funções bastante complexo, uma vez que não cumprem um papel específico no metabolismo, no crescimento e na divisão celular do organismo produtor. Estão, por outro lado, intrinsecamente relacionados aos mecanismos de defesa do vegetal em face dos mais distintos fatores de pressão de seleção do ambiente, em especial aqueles voltados contra o ataque de herbívoros ${ }^{14}$.

Diversos componentes químicos provenientes do metabolismo secundário de vegetais, como alcaloides, esteroides, terpenoides e fenólicos, desempenham um importante papel sobre vários insetos vetores de doenças ${ }^{7}$, seja inibindo a alimentação, reduzindo a oviposição, seja prejudicando o crescimento de suas formas imaturas ou atraindo inimigos naturais ${ }^{6,14}$.

É importante destacar que a ação larvicida de um extrato vegetal ou óleo essencial pode variar de maneira significativa de acordo com a espécie de inseto a ser desafiada com tais produtos, a espécie e a idade da planta, a região geográfica de sua ocorrência (existência de quimiotipos), as estruturas utilizadas e a polaridade do solvente utilizado durante o processo de obtenção ${ }^{16}$.

\section{CONSIDERAÇÕES FINAIS}

A busca por novas estratégias que visem ao controle de insetos transmissores de doenças tem sido amplamente reforçada devido ao aumento de sua resistência aos inseticidas sintéticos tradicionalmente utilizados, bem como aos prejuízos ambientais advindos de seu uso indiscriminado ${ }^{5}$. Nessa perspectiva, produtos vegetais têm ganhado cada vez mais destaque em virtude da grande variedade e da quantidade de substâncias que exibem tal propriedade ${ }^{82}$.

A prospecção de extratos vegetais e óleos essenciais que exibem atividade larvicida contra Ae. aegypti, Ae. albopictus e outras espécies de culicídeos de importância médica, como Culex quinquefasciatus e Anopheles darlingi, tem sido fortemente influenciada pelo aumento da prevalência/incidência das doenças por eles veiculadas, além da baixa eficiência das formulações dos produtos tradicionalmente destinados ao controle das formas aladas desses insetos ${ }^{83}$.

\section{REFERÊNCIAS}

1. Ghosh A, Chowdhury N, Chandra G. Plant extracts as potential mosquito larvicides. Indian J Med Res. 2012 May; 135(5) :581-598. PubMed PMID: 22771587

2. Klempner MS, Unnasch TR, Hu LT. Taking a bite out of vector-transmitted infectious diseases. N Engl J Med. 2007; 356:2567-2569. doi: 10.1056/ NEJMp078081.

3.Jirakanjanakit N, Rongnoparut $\mathrm{P}$, Saengtharatip S, Chareonviriyaphap T, Duchon S, Bellec C, et al. Insecticide Susceptible / Resistance Status in Aedes (Stegomyia) aegypti and Aedes (Stegomyia) albopictus (Diptera:Culicidae) in Thailand during 2003 - 2005. J Econ Entomol. 2007 Apr; 100(2): 545-550.

4. Sarwar M, Ahmad N, Toufiq M. Host-plant-resistance-relationships in chickpea (Cicer arietinum L.) against gram pod borer (Helicoverpa armigera Hubner). Pak J Bot. 2009; 41(6): 3047-3052.

5. Jantan IB, Yalvema MF, Ahmad NW, Jamal JA. Insecticidal activities of the leaf oils of eight Cinnamomum species against Aedes aegypti and Aedes albopictus. Pharm Biol. 2005; 43(6):526-532. doi: https://doi. org/10.1080/13880200500220771.
6. Isman MB. Botanical Insecticides, Deterrents, and Repellents in Modern Agriculture and an Increasingly Regulated World. Annu Rev Entomol. 2006; 51(1):45-66. doi: 10.1146/annurev.ento.51.110104.151146.

7. Shaalan E, Canyon D, Faried MW, Abdel-Wahab H, Mansour A. A review of botanical phytochemicals with mosquitocidal potential. Environ Int. 2005 Oct; 31(8):1149-1166. doi: 10.1016/j.envint.2005.03.003.

8. Zhu J, Zeng X, O `Neal M, Schultz G, Tucker B, Coats J, et al. Mosquito larvicidal activity of botanical-based mosquito repellents. J Am Mosq Control Assoc. 2008 Mar; 24(1):161-168.

9. Sharma P, Mohan L, Srivastava CN. Amaranthus oleracea and Euphorbia hirta: Natural potential larvicidal agents against the urban Indian malaria vector, Anopheles stephensi Liston (Diptera: Culicidae). Parasitol Res. 2009 Dec; 106(1): 171-176. doi: 10.1007/s00436-009-1644-1

10. Cheng SS, Chang HT, Chang ST, Tsai KH, Chen WJ. Bioactivity of selected plant essential oils against the yellow fever mosquito Aedes aegypti larvae. Bioresour Technol. 2003 Aug; 89(1): 99-102. PubMed PMID: 12676507.

11. Martins VEP, Alencar CHM, Facó PEG, Dutra RF, Alves CR, Pontes RJS, Guedes 
MIF. Distribuição espacial e características dos criadouros de Aedes albopictus e Aedes aegypti em Fortaleza, Estado do Ceará. Rev Soc Bras Med Trop. 2010 JanFev; 43(1): 73-77. doi: http://dx.doi.org/10.1590/\$0037-86822010000100016.

12. Saito ML, Scramin S. Plantas aromáticas e seu uso na agricultura. Jaguariúna: Embrapa Meio Ambiente; 2000. 48 p.

13. Cosimi S, Rossi E, Cioni PL, Canale A. Bioactivity and qualitative analysis of some essential oils from Mediterranean plants against stored-product pests, Evaluation of repellency against Sitophilus zeamais Motschulsky, Cryptolestes ferrugineus Stephens and Tenebrio molitor L. J Stored Prod Res. 2009 Apr; 45(2): 125-132. doi: https://doi.org/10.1016/j.jspr.2008.10.002.

14. Simões CMO, Schenkel EP, Mello JCP, Mentz LA, Petrovick PR. Farmacognosia: do produto natural ao medicamento. Porto Alegre: UFSC/UFRGS; 2017.

15. Halfeld-Vieira BA, Marinho-Prado JS, Nechet KL, Morandi MAB, Bettiol W. Defensivos Agrícolas Naturais: uso e perspectivas. Brasilia: EMBRAPA; 2016

16. Cheng SS, Huang CG, Chen YJ, Yu JJ, Chen WJ, Chang ST. Chemical compositions and larvicidal activities of leaf essential oils from two eucalyptus species. Bioresou Technol. 2009 Jan; 100(1): 452-456. doi: 10.1016/j. biortech.2008.02.038.

17. Govindarajan M. Evaluation of Andrographis paniculata Burm. f (Family:Acanthaceae) extracts against Culex quinquefasciatus (Say.) and Aedes aegypti (Linn.) (Diptera:Culicidae). Asian Pac J of Trop Med. 2011 Mar; 4(3):176181. doi: 10.1016/S1995-7645(11)60064-3.

18. Renugadevi G, Ramanathan T, Shanmuga PR, Thirunavukkarasu P. Studies on effects of Andrographis paniculata (Burm.f.) and Andrographis lineata nees (Family: Acanthaceae) extracts against two mosquitoes Culex quinquefasciatus (Say.) and Aedes aegypti (Linn.). Asian Pac J Trop Med. 2013 Mar; 6(3):176-179. doi: 10.1016/S1995-7645(13)60019-X.

19. Dias CN, Moraes DFC. Essential oils and their compounds as Aedes aegypti L. (Diptera: Culicidae) larvicides: Review. Parasitol Res. 2014 Feb; 113(2): 565-592. doi: 10.1007/s00436-013-3687-6.

20. Yousaf A, Zuharah WF. Lethal response of the dengue vectors to the plant extracts from family Anacardiaceae. Asian Pac J Trop Biomed. 2015 Oct; 5(10): 812-818. doi: https://doi.org/10.1016/j.apjtb.2015.05.016.

21. Aciole SDG, Piccoli CF, Duque LJE, Costa EV, Navarro-Silva MA, Marques FA, Maia B, Pinheiro MLB, Rabelo MT. Insecticidal activity of three species of Guatteria (Annonaceae) against Aedes aegypti (Diptera: Culicidae). Rev Colomb Entomol. 2011 Jul-Dec; 37(2):262-268.

22. Kumar S, Mishra M, Wahab N, Warikoo R. Larvicidal, repellent, and irritant potential of the seed-derived essential oil of Apium graveolens against dengue vector, Aedes aegypti L. (Diptera: Culicidae). Front Public Health. 2014 Sep; 2:147. doi: 10.3389/fpubh.2014.00147.

23. Govindarajan M, Benelli G. Eco-friendly larvicides from Indian plants: Effectiveness of lavandulyl acetate and bicyclogermacrene on malaria, dengue and Japanese encephalitis mosquito vectors. Ecotoxicol Environ Saf. 2016 Nov; 133:395-402. doi: 10.1016/j.ecoenv.2016.07.035.

24. Kabir KE, Choudhary MI, Ahmed S, Tariq RM. Growth-disrupting, larvicidal and neurobehavioral toxicity effects of seed extract of Seseli diffusum against Aedes aegypti (L.) (Diptera: Culicidae). Ecotoxicol Environ Saf. 2013 Apr; 90: 52-60. doi: 10.1016/j.ecoenv.2012.12.028.

25. Castillo FD, Cardona SMM, Medina MC, González YP, Estrada HG. Actividad larvicida de extractos etanólicos de Tabernaemontana cymosa y Trichilia hirta sobre larvas de estadio III y IV de Aedes aegypti (Diptera: Culicidae). Rev Cuba Plantas Med. 2012; 17(3):256-267.

26. Fazal S, Manzoor F, Latif AA, Munir N, Izza PM. Larvicidal activities of five essential oils against Aedes aegypti (L.) larvae (diptera: Culicidae). Asian J Chem. 2013; 25(18): 10212-10216.

27. Furtado RF, Lima MGA, Neto MA, Bezerra JNS, Silva MGV. Atividade larvicida de óleos essenciais contra Aedes aegypti L. (Diptera: Culicidae). Neotrop Entomol. 2005; 34(5): 843-847. doi: http://dx.doi.org/10.1590/S1519$566 \times 2005000500018$

28. Leyva M, Tacoronte JE, Marquetti MC, Scull R, Montada D, Rodríguez $\mathrm{Y}$, et al. Actividad insecticida de aceites esenciales de plantas en larvas de Aedes aegypti (Diptera: Culicidae). Rev Cubana Med Trop. 2008 Jan-Abr; 60(1): 78-82.

29. Tennyson S, Ravindran KJ, Arivoli S. Bioefficacy of botanical insecticides against the dengue and chikungunya vector Aedes aegypti (L.) (Diptera: Culicidae). Asian Pac J Trop Biomed. 2012; 2(3):1842-1844. doi: https://doi. org/10.1016/S2221-1691(12)60505-X.

30. Lima WP, Neto FC, Macoris MLG, Zuccari DAPC, Dibo MR. Estabelecimento de metodologia para alimentação de Aedes aegypti (Diptera:Culicidae) em camundongos swiss e avaliação da toxicidade e do efeito residual do óleo essencial de Tagetes minuta L (Asteraceae) em populações de Aedes aegypti. Rev Soc Bras Med Trop. 2009 Dez; 42(6):638-641. doi: http://dx.doi.org/10.1590/ S0037-86822009000600005.

31. Dharmagadda VSS, Naik SN, Mittal PK, Vasudevan P. Larvicidal activity of Tagetes patula essential oil against three mosquito species. Bioresour Tech. 2005; 96(11): 1235-1240. doi: 10.1016/j.biortech.2004.10.020.

32. Rajasekaran A, Duraikannan G. Larvicidal activity of plant extracts on Aedes aegypti L. Asian Pac J Trop Biomed. 2012; 2(3):1578-1582. doi: https://doi. org/10.1016/S2221-1691(12)60456-0.

33. Silva HHG, Geris R, Filho ER, Rocha C, Silva IG. Larvicidal activity of oilresin fractions from the Brazilian medicinal plant Copaifera reticulata Ducke (Leguminosae-Caesalpinoideae) against Aedes aegypti (Diptera, Culicidae). Rev Soc Bras Med Trop. 2007; 40(3): 264-267. doi: http://dx.doi.org/10.1590/ S0037-86822007000300002.

34. Wahyuni D. New bioinsecticide granules toxin from extract of Papaya (Carica Papaya) seed and leaf modified against Aedes aegypti larvae. Procedia Environ Sci. 2015; 23:323-328. doi: https://doi.org/10.1016/j.proenv.2015.01.047

35. Ishak AR, Dom NC, Hussain H, Sabri NH. Biolarvacidal Potential of Ipomoea Cairica extracts against key dengue vectors. Procedia Soc BehavSci. 2014; 153:180-188. doi: https://doi.org/10.1016/j.sbspro.2014.10.052.

36. Ali MS, Ravikumar S, Beula JM. Bioactivity of seagrass against the dengue fever mosquito Aedes aegypti larvae. Asian Pac J Trop Biomed. 2012 Jul; 2(7): 570-573. doi: 10.1016/S2221-1691(12)60099-9.

37. Lima MGA, Maia ICC, Sousa BD, Morais SM, Freitas SM. Effect of stalk and leaf extracts from euphorbiaceae species on Aedes aegypti (Diptera, Culicidae) larvae. Rev Inst Med Trop Sao Paulo. 2006 Jul-Ago; 48(4):211-214. PubMed PMID: 17119677.

38. Dória GAA, Silva WJ, Carvalho GA, Alves PB, Cavalcanti SCH. A study of the larvicidal activity of two Croton species from northeastern Brazil against Aedes aegypti. Pharm Biol. 2010 Jun; 48(6):615-620. doi: 10.3109/13880200903222952.

39. Trindade FTT, Stabeli RG, Pereira AA, Facundo VA, Silva AA. Copaifera multijuga ethanolic extracts, oil-resin, and its derivatives display larvicidal activity against Anopheles darlingi and Aedes aegypti (Diptera: Culicidae). Braz J Pharmacog, 2013 Maio-Jun; 23(3):464-470. doi: http://dx.doi.org/10.1590/ S0102-695X2013005000038.

40. Pluempanupat S, Kumrungsee N, Pluempanupat WNK, Chavasiri W, Bullangpoti V, Koul O. Laboratory evaluation of Dalbergia oliveri (Fabaceae: Fabales) extracts and isolated isoflavonoids on Aedes aegypti (Diptera: Culicidae) mosquitoes. Ind Crops Prod. 2013 Jan; 44: 653-658. doi: https://doi. org/10.1016/j.indcrop.2012.09.006

41. Kolli GR, Balakrishnan, Vijayan, Sundararajan R. Evaluation of larvicidal activity of Pongamia pinnata extracts against three mosquito vectors. Asian Pac J Trop Biomed. 2013 Nov; 3(11):853-858. doi: 10.1016/S2221-1691(13)601689. 
42. Silva OS, Silva FC, Barros FMC, Silva JLR, Bordignon SAL, Eifler-Lima VL, von Poser GL, Prophiro JS. Larvicidal and growth-inhibiting activities of extract and benzopyrans from Hypericum polyanthemum (Guttiferae) against Aedes aegypti (Diptera: Culicidae). Ind Crops Prod. 2013 Feb; 45:236-239. doi: https:// doi.org/10.1016/j.indcrop.2012.12.025.

43. Kalaivani K, Senthil-Nathan S, Murugesan AG. Biological activity of selected Lamiaceae and Zingiberaceae plant essential oils against the dengue vector Aedes aegypti L. (Diptera: Culicidae). Parasitol Res. 2012 Mar; 110(3):12611268. doi: 10.1007/s00436-011-2623-x.

44. Kumar S, Wahab N, Warikoo R. Bioefficacy of Mentha piperita essential oil against dengue fever mosquito Aedes aegypti L. Asian Pac J Trop Biomed. 2011 Apr; 1(2): 85-88. doi: 10.1016/S2221-1691(11)60001-4.

45. Lima TC, Silva TKM, Silva FL, Barbosa JM Filho, Marques MOM, Santos RLC, et al. Larvicidal activity of Mentha $x$ villosa Hudson essential oil, rotundifolone and derivatives. Chemosphere. 2014 Jun; 104:37-43. doi: 10.1016/j. chemosphere.2013.10.035.

46. Govindarajan M, Kadaikunnan S, Alharbi NS, Benelli G. Acute toxicity and repellent activity of the Origanum scabrum Boiss. \& Heldr. (Lamiaceae) essential oil against four mosquito vectors of public health importance and its biosafety on non-target aquatic organisms. Environ Sci Pollut Res. 2016 Nov; 23(22):23228-23238.

47. Torres RC, Garbo AG, Walde RZML. Larvicidal activity of Persea americana Mill. against Aedes aegypti. Asian Pac J Trop Med. 2014 Sep; 7(1):167-170. doi: 10.1016/S1995-7645(14)60225-X.

48. Govindarajan M. Larvicidal and repellent activities of Sida acuta Burm. F. (Family: Malvaceae) against three important vector mosquitoes. Asian Pac J Trop Med. 2010; 3(9): 691-695. doi: https://doi.org/10.1016/S1995-7645(10)601678.

49. Lucia AL, Licastro S, Zerba E, Masuh H. Yield, chemical composition, and bioactivity of essential oils from 12 species of Eucalyptus on Aedes aegypti larvae. Entomol Exp App. 2008 Sep; 129(1): 107-114. doi: https://doi. org/10.1111/j.1570-7458.2008.00757.x

50. Dias CN, Alves LPL, Rodrigues KAF, Brito MCA, Rosa CS, Amaral FMM, et al. Chemical composition and larvicidal activity of essential oils extracted from Brazilian legal Amazon plants against Aedes aegypti L. (Diptera: Culicidae). Evid Based Complement Alternat Med. 2015. doi: 10.1155/2015/490765.

51. Fayemiwo KA, Adeleke MA, Okoro OP, Awojide SH, Awoniyi IO. Larvicidal efficacies and chemical composition of essential oils of Pinus sylvestris and Syzygium aromaticum against mosquitoes. Asian Pac J Trop Biomed. 2014 Jan; 4(1): 30-34. doi: 10.1016/S2221-1691(14)60204-5.

52. Govindarajan M, Rajeswary M, Benelli G. Ecotoxicology and Environmental Safety Chemical composition, toxicity and non-target effects of Pinus kesiya essential oil : An eco-friendly and novel larvicide against malaria, dengue and lymphatic fi lariasis mosquito vectors. Ecotoxicol Environ Saf. 2016 Jul; 129: 85-90. doi: 10.1016/j.ecoenv.2016.03.007.

53. Santana HT, Trindade F, Stabeli RG, Silva AAE, Militão JSLT, Facundo VA. Essential oils of leaves of piper species display larvicidal activity against the dengue vector, Aedes aegypti (diptera: Culicidae). Rev bras plantas med. 2015 Jan-Mar; 17(1):105-111. doi: http://dx.doi.org/10.1590/1983-084X/13_052.

54. Raj GA, Chandrasekaran M, Krishnamoorthy S, Jayaraman M, Venkatesalu $V$. Phytochemical profile and larvicidal properties of seed essential oil from Nigella sativa L. (Ranunculaceae), against Aedes aegypti, Anopheles stephensi and Culex quinquefasciatus (Diptera:Culicidae). Parasitol Res. 2015 Sep; 114(9): 3385-3391. doi: 10.1007/s00436-015-4563-3.

55. Warikoo R, Ray A, Sandhu JK, Samal R, Wahab N, Kumar S. Larvicidal and irritant activities of hexane leaf extracts of Citrus sinensis against dengue vector Aedes aegypti L. Asian Pac J Trop Biomed. 2012 Feb; 2(2):152-155. doi: 10.1016/S2221-1691(11)60211-6.

56. Rajkumar S, Jebanesan A. Chemical composition and larvicidal activity of leaf essential oil from Clausena dentata (Willd) M. Roam. (Rutaceae) against the chikungunya vector, Aedes aegypti Linn. (Diptera: Culicidae). J Asia-Pac Entomol. 2010; 13(2):107-109. doi: https://doi.org/10.1016/j.aspen.2010.02.001.

57. Manjari MS, Karth S, Ramkumar G, Muthusamy R, Natarajan D, Shivakumar MS. Chemical composition and larvicidal activity of plant extracts from Clausena dentata (Willd) (Rutaceae) against dengue, malaria, and filariasis vectors. Parasitol Res. 2014 Jul; 113(7):2475-2481. doi: 10.1007/s00436-014-3896-7.

58. Krishnamoorthy SCM, Raj GA, Jayaraman M, Venkatesalu V. Identification of chemical constituents and larvicidal activity of essential oil from Murraya exotica L. (Rutaceae) against Aedes aegypti, Anopheles stephensi and Culex quinquefasciatus (Diptera: Culicidae). Parasitol Res. 2015 May; 114(5):18391845. doi: 10.1007/s00436-015-4370-x.

59. Pitasawat B, Champakaew D, Choochote W, Jitpakdi A, Chaithong U, Kanjanapothi D, et al. Aromatic plant-derived essential oil: An alternative larvicide for mosquito control. Fitoterapia. 2007 Apr; 78(3): 205-210. doi: 10.1016/j.fitote.2007.01.003.

60. Intirach J, Junkum A, Lumjuan N, Chaithong U, Jitpakdi A, Riyong D, et al. Antimosquito property of Petroselinum crispum (Umbellifereae) against the pyrethroid resistant and susceptible strains of Aedes aegypti (Diptera: Culicidae). Environ Sci Pollut Res Int. 2016 Dec; 23(23):23994-24008. doi: 10.1007/s11356-016-7651-8

61. Govindarajan M, Rajeswary M, Arivoli S, Tennyson S, Benelli G. Larvicidal and repellent potential of Zingiber nimmonii (J. Graham) Dalzell (Zingiberaceae) essential oil: an eco-friendly tool against malaria, dengue, and lymphatic filariasis mosquito vectors? Parasitol Res. 2016; 115(5):1807-1816. doi: 10.1007/s00436-016-4920-x.

62. El-Sheikh TMY, Al-Fifi ZIA, Alabboud MA. Larvicidal and repellent effect of some Tribulus terrestris L., (Zygophyllaceae) extracts against the dengue fever mosquito, Aedes aegypti (Diptera: Culicidae). J Saudi Chem Soc. 2016; 20(1):1319. doi: https://doi.org/10.1016/j.jscs.2012.05.009

63. Liu XC, Liu Q, Zhou L, Liu ZL. Evaluation of larvicidal activity of the essential oil of Allium macrostemon Bunge and its selected major constituent compounds against Aedes albopictus (Diptera: Culicidae). Parasit Vectors. 2014; 7: 184-188. doi: 10.1186/1756-3305-7-184.

64. Benelli G, Flamini G, Fiore G, Cioni PL, Conti B. Larvicidal and repellent activity of the essential oil of Coriandrum sativum L. (Apiaceae) fruits against the filariasis vector Aedes albopictus Skuse (Diptera: Culicidae). Parasitol Res. 2013 Mar; 112(3):1155-1161. doi: 10.1007/s00436-012-3246-6.

65. Conti B, Canale A, Bertoli A, Gozzini F, Pistelli L. Essential oil composition and larvicidal activity of six Mediterranean aromatic plants against the mosquito Aedes albopictus (Diptera: Culicidae). Parasitol Res. 2010; 107(6):1455-1461. doi: 10.1007/s00436-010-2018-4

66. Bedini S, Flamini G, Cosci F, Ascrizzi R, Benelli G, Conti B. Cannabis sativa and Humulus lupulus essential oils as novel control tools against the invasive mosquito Aedes albopictus and fresh water snail Physella acuta. Ind Crops Prod. 2016 Jul; 85:318-323. doi: https://doi.org/10.1016/j.indcrop.2016.03.008

67. Liu ZL, He Q, Chu SS, Wang CF, Du SS, Deng ZW. Essential oil composition and larvicidal activity of Saussurea lappa roots against the mosquito Aedes albopictus (Diptera: Culicidae). Parasitol Res. 2012 Jun; 110(6): 2125-2130. doi: 10.1007/s00436-011-2738-0.

68. Giatropoulos A, Pitarokili D, Papaioannou F, Papachristos DP, Koliopoulos G, Emmanouel $\mathrm{N}$, et al. Essential oil composition, adult repellency and larvicidal activity of eight Cupressaceae species from Greece against Aedes albopictus (Diptera: Culicidae). Parasitol Res. 2013 Mar; 112(3):1113-1123. doi: 10.1007/ s00436-012-3239-5

69. Mandal S. Exploration of larvicidal and adult emergence inhibition activities of Ricinus communis seed extract against three potential mosquito vectors in Kolkata, India. Asian Pac J Trop Med. 2010 Aug; 3(8):605-609. doi: https://doi. org/10.1016/S1995-7645(10)60147-2.

70. Li W, Huang C, Wang K, Fu J, Cheng D, Zhang Z. Laboratory evaluation of 
aqueous leaf extract of Tephrosia vogelii against larvae of Aedes albopictus (Diptera: Culicidae) and non-target aquatic organisms. Acta Trop. 2015 Jun; 146: 36-41. doi: 10.1016/j.actatropica.2015.02.004.

71. Govindarajan M, Sivakumar R, Rajeswary M, Veerakumar K. Mosquito larvicidal activity of thymol from essential oil of Coleus aromaticus Benth. against Culex tritaeniorhynchus, Aedes albopictus, and Anopheles subpictus (Diptera: Culicidae). Parasitol Res. 2013 Nov; 112(11):3713-3721. doi: 10.1007/ s00436-013-3557-2.

72. Conti B, Benelli G, Flamini G, Cioni PL, Profeti R, Ceccarini L, et al. Larvicidal and repellent activity of Hyptis suaveolens (Lamiaceae) essential oil against the mosquito Aedes albopictus Skuse (Diptera: Culicidae). Parasitol Res. 2012 May; 110(5): 2013-2021. doi: 10.1007/s00436-011-2730-8.

73. Govindarajan M, Sivakumar R, Rajeswary M, Yogalakshmi K. Chemical composition and larvicidal activity of essential oil from Ocimum basilicum (L.) against Culex tritaeniorhynchus, Aedes albopictus and Anopheles subpictus (Diptera: Culicidae). Exp Parasitol. 2013 May; 134(1): 7-11. doi: 10.1016/j. exppara.2013.01.018.

74. Sumitha KV, Thoppil JE. Larvicidal efficacy and chemical constituents of O. gratissimum L. (Lamiaceae) essential oil against Aedes albopictus Skuse (Diptera: Culicidae). Parasitol Res. 2016 Feb; 115(2):673-680. doi: 10.1007/ s00436-015-4786-3

75. Mathew J, Thoppil JE. Chemical composition and mosquito larvicidal activities of Salvia essential oils. Pharm Biol. 2011 May; 49(5):456-463. doi: 10.3109/13880209.2010.523427.

76. Cheng SS, Liu JY, Huang CG, Hsui YR, Chen WJ, Chang ST. Insecticidal activities of leaf essential oils from Cinnamomum osmophloeum against three mosquito species. Bioresour Technol. 2009 Jan; 100(1):457-464. doi: 10.1016/j. biortech.2008.02.030.

77. Francine TN, Cabral BNP, Anatole PC, Bruno MM, Pauline N, Jeanne NY.
Larvicidal activities of hydro-ethanolic extracts of three Cameroonian medicinal plants against Aedes albopictus. Asian Pac J Trop Biomed. 2016 Nov; 6(11):931936. doi: https://doi.org/10.1016/j.apjtb.2016.09.004.

78. Koutsaviti K, Giatropoulos A, Pitarokili D, Papachristos D, Michaelakis A, Tzakou O. Greek Pinus essential oils: larvicidal activity and repellency against Aedes albopictus (Diptera: Culicidae). Parasitol Res. 2015 Feb; 114(2):583-592. doi: 10.1007/s00436-014-4220-2.

79. Giatropoulos A, Papachristos DP, Kimbaris A, Koliopoulos G, Polissiou MG, Emmanouel N, Michaelakis A. Evaluation of bioefficacy of three Citrus essential oils against the dengue vector Aedes albopictus (Diptera: Culicidae) in correlation to their components enantiomeric distribution. Parasitol Res. 2012 Dec; 111(6):2253-2263. doi: 10.1007/s00436-012-3074-8.

80. Conti B, Leonardi M, Pistelli L, Profeti R, Ouerghemmi I, Benelli G. Larvicidal and repellent activity of essential oils from wild and cultivated Ruta chalepensis L. (Rutaceae) against Aedes albopictus Skuse (Diptera: Culicidae), an arbovirus vector. Parasitol Res. 2013 Mar; 112(3):991-999. doi: 10.1007/s00436-0123221-2.

81. Liu XC, Dong HW, Zhou L, Du SS, Liu ZL. Essential oil composition and larvicidal activity of Toddalia asiatica roots against the mosquito Aedes albopictus (Diptera: Culicidae). Parasitol Res. 2013 Mar; 112(3):1197-1203. doi: 10.1007/s00436-012-3251-9.

82. Kamiabi F, Jaal Z, Keng CL. Bioefficacy of crude extracts of Cyperus aromaticus (Family:Cyperaceae) cultured cells, against Aedes aegypti and Aedes albopictus mosquitoes. Asian Pac J Trop Biomed. 2013 Oct; 3(10):767-775. doi: 10.1016/ S2221-1691(13)60153-7.

83. Beula JM, Ravikumar S, Ali MS. Mosquito larvicidal efficacy of seaweed extracts against dengue vector of Aedes aegypti. Asian Pac J Trop Med. 2011 Oct; (2, Suppl): S143-S146. doi: https://doi.org/10.1016/S2221-1691(11)601433.

\section{Como citar este artigo/How to cite this article:}

Viana GA, Sampaio CG, Martins VEP. Produtos naturais de origem vegetal como ferramentas alternativas para o controle larvário de Aedes aegypti e Aedes albopictus. J Health Biol Sci. 2018 Out-Dez; 6(4):449-462. 